\title{
Obesity and Metabolic Syndrome, Too Big of an Enemy to Be Just Fought with a Scalpel
}

\author{
Joost P. H. Drenth ${ }^{1} \cdot$ Richard P. G. ten Broek ${ }^{2}$. Cornelius J. H. M. van Laarhoven ${ }^{2}$
}

Published online: 27 November 2019

(C) The Author(s) 2019

Keywords Obesity $\cdot$ Metabolic syndrome $\cdot$ Surgery $\cdot$ Integrative medicine

Obesity defined as a body mass index (BMI) $>30 \mathrm{~kg} / \mathrm{m}^{2}$ has become a global epidemic. The prevalence is increasing and currently, $30 \%$ of the western population are obese, while $15 \%$ has severe obesity $\left(\mathrm{BMI}>35 \mathrm{~kg} / \mathrm{m}^{2}\right)[1]$.

Obesity puts patients at an increased lifetime risk of a host of chronic disorders and is closely linked with emergence of diabetes, gallstones, non-alcoholic fatty liver disease, chronic kidney failure, cardiovascular diseases, many types of cancers, and osteoarthritis (Fig. 1a).

Due to the rise of obesity, more than $50 \%$ of the population aged over 50 will suffer from one or more chronic diseases. The average age span obese people have to cope with a chronic disease has increased to 30 years (males) and 40 years (females). The relative mortality risk in obese is increased to 2.7 , resulting in a reduced life expectancy with as much as 8 years [2]. According to the World Health Organization, obesity is directly related to $5 \%$ of all deaths worldwide translating in 2.8 million out of 59 million annual total global deaths [3]. The economical global impact of obesity alone is 2.0 trillion US dollar, equal to $2.8 \%$ of the worldwide gross domestic product [4]. Obesity has now become the biggest threat to public health. For example, whereas the lifetime risk of incurring lung cancer is $\sim 16 \%$ in young smokers, the likelihood of overweight adolescents to develop metabolic syndrome is almost $100 \%$. In the USA, obesity has already surpassed smoking in terms of quality-adjusted life years lost.

The consequence of obesity and the development of the metabolic syndrome is that patients inevitably will develop

Cornelius J. H. M. van Laarhoven

kees.vanlaarhoven@radboudmc.nl

1 Department of Gastroenterology and Hepatology, Radboud University Medical Center, Nijmegen, The Netherlands

2 Department for Surgery, Radboud University Medical Center, P.O. Box 9101, 6500 HB Nijmegen, The Netherlands indications for surgical therapy. Obese patients have a high lifetime risk to receive surgical procedures such as cholecystectomy, vascular stenting, coronary bypass surgery, and/or joint replacements. Many obese patients will develop an indication for bariatric surgery such as the gastric sleeve and Roux-en-Y gastric bypass. Bariatric procedures result in long-term weight loss, improved diabetic regulation and decreased overall mortality [5].

While this success of bariatric surgery may be hailed as a victory in the fight against obesity, there is an important flip side. If we continue to accept a $15 \%$ prevalence of severe obesity (the threshold for bariatric surgery in international guidelines), our healthcare system will not be able to cope with the growing healthcare consumption [5]. Facing a world population growth to 8.5 billion people in 2030, 12 years from now, 1.3 billion people will develop an indication for bariatric surgery.

The question arises whether this is a desirable development. The real question here is whether we should focus on the surgical palliation of (the consequences of) obesity or that we divert our resources and energy to its' root cause. The metabolic syndrome kills, and the worst is yet to come. Healthcare redesign, focusing on care innovation rather than technical innovation alone, is vital. Whereas the strategy of technical innovations has been effective in communicable diseases (e.g., sanitation, antibiotics, and antiviral therapies) and becoming more effective in oncology (chemoradiotherapy and immune therapy), the metabolic syndrome requires a totally different approach.

Apart from the mediocre long-term cost-effectiveness of bariatric surgery and the negative long-term cost-benefit outcome of symptomatic treatment of the metabolic syndrome as a whole, other questions arise. The exploding healthcare consumption is eroding the sustainability of the traditional disease care system we live in. We, as surgeons, can no longer continue to merely "picking apples from the tree" (Fig. 1b), and 
Fig. 1 a "Tree of life." $\mathbf{b}$ "Tree of life." Surgical picking apples from the tree. (NAF, nonalcoholic fatty); Copyright CJHM van Laarhoven $\odot /$ Gloedcommunications $^{\mathrm{TM}}$

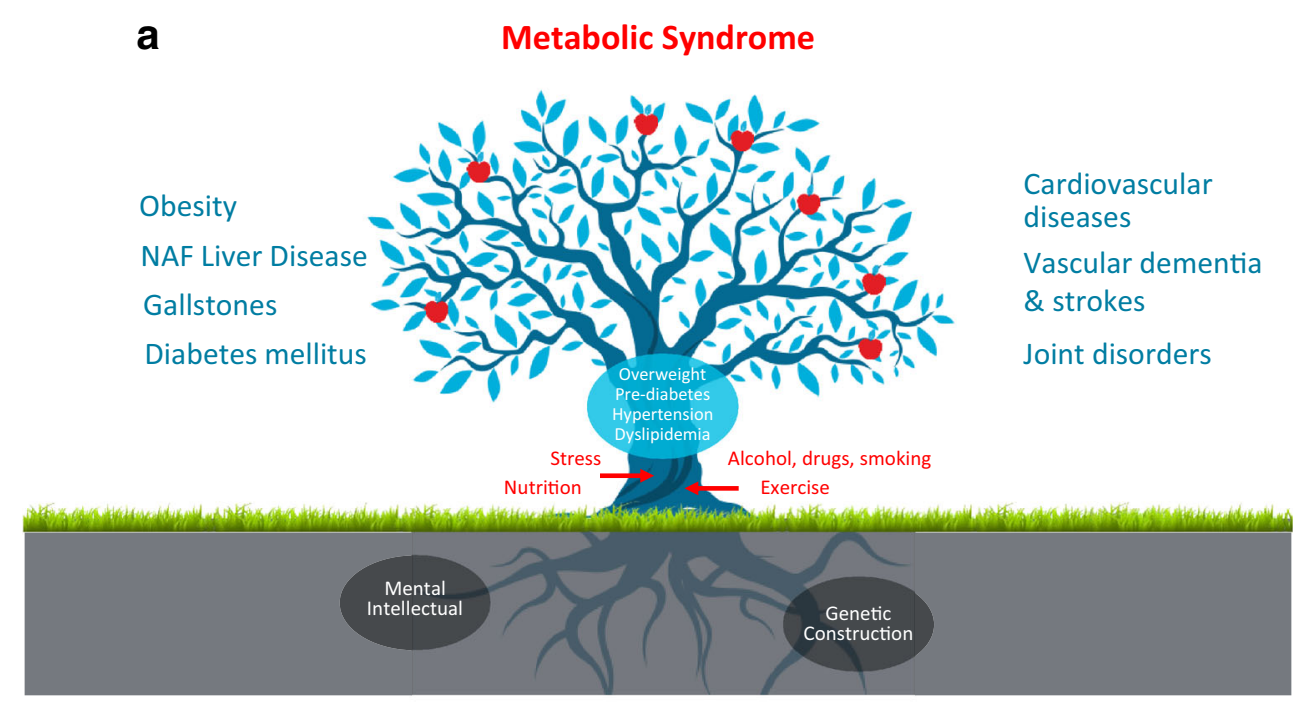

b

Metabolic Syndrome

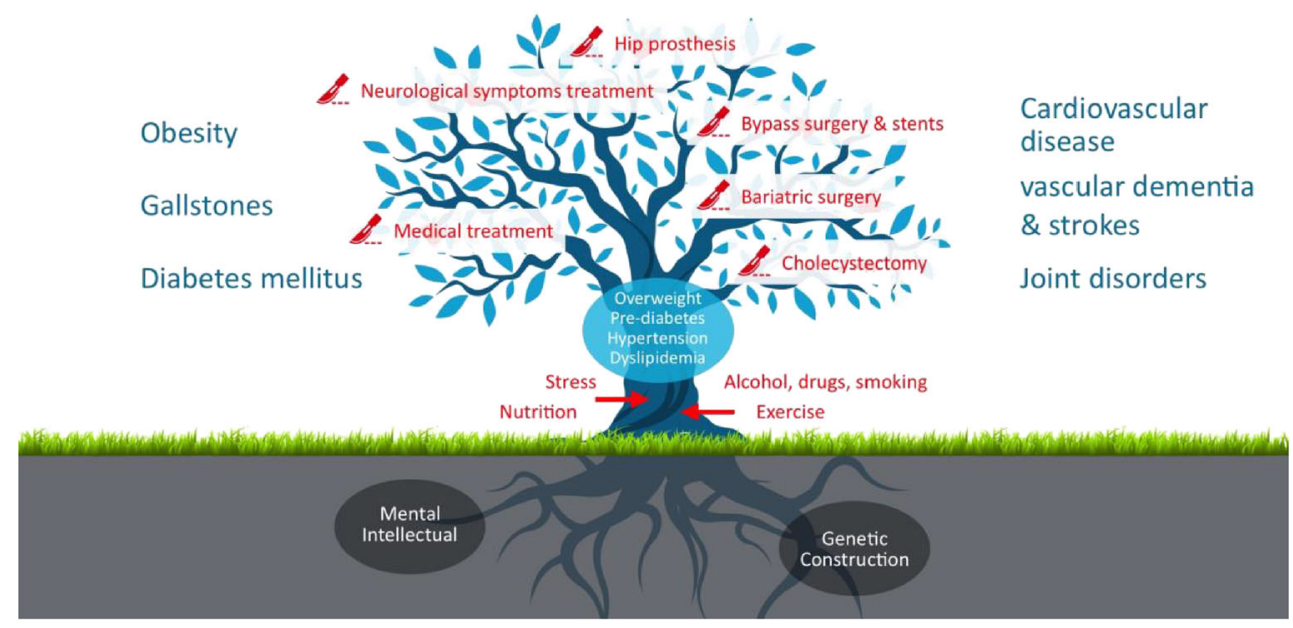

look away when we realize that more is needed to prevent subsequent diseases in the unhealthy future decades of our patients.

We also need to address the philosophical/ethical question: do we, as society accepts the continuous metabolic degeneration of mankind is an acceptable, but inevitable evolution? This clearly broadens the perspective of the problem. We live in a very "obesogenic" environment and patients are regarded as victims, while they are often unaware of how their own behavior contributes to imminent diseases [6]. And although self-responsible, we are unable to readjust our lifestyle and simply allow the food-, alcohol-, and tobacco industries to fuel this "obesogenic" world.

Despite the era of technical and medical innovations and despite the fact that healthcare delivery, in Western Europe, has never been of such a high standard, we have never been further off from "healthy aging."

We as medical professionals can make a difference. Realizing that the metabolic syndrome is a silent killer, healthcare redesigning, focusing on care innovation rather than technical innovation alone, is vital [7]. Apart from symptomatic intervention treatments, a non-surgical approach towards patient self-awareness of lifestyle and healthy attitudes is needed to provide a decrease in healthcare consumption towards a more sustainable global healthcare system.

Therefore, obese patients, presenting with one of the metabolic syndrome related diseases should be considered both for medical or surgical treatment and integrative approach by offering lifestyle and health promotion interventions to all surgical patients with metabolic syndrome-related diseases. 
Multidisciplinary lifestyle outpatient clinics, as an integrated part of the surgical treatment, will help patients to prevent them from having a second or third health event. Sustainable lifestyle changes can only be reached when patients, surgeons, endocrinologists, lifestyle coaches, dietitians, psychologists, and many other healthcare providers team up. We, as physicians, have the knowledge and authority to influence both patients, population, and society to let them understand that prevention of the metabolic syndrome is vital in maintaining a sustainable healthcare system.

A complementary, holistic approach is needed combining symptomatic-preferable conservative - treatment of metabolic syndrome-related diseases, with a preventive approach to reduce or prevent future metabolic deterioration. The metabolic syndrome is too big of an enemy to be just fought with a scalpel.

Author Contributions $\mathrm{CvL}$ had the original idea and wrote the initial draft. RtB critically reviewed the manuscript. JD critically reviewed the manuscript, and all authors approve the final version of the manuscript.

\section{Compliance with Ethical Standards}

Conflict of Interest The authors declare that they have no conflict of interest.

Open Access This article is distributed under the terms of the Creative Commons Attribution 4.0 International License (http:// creativecommons.org/licenses/by/4.0/), which permits unrestricted use, distribution, and reproduction in any medium, provided you give appropriate credit to the original author(s) and the source, provide a link to the Creative Commons license, and indicate if changes were made.

\section{References}

1. Ng M, Fleming T, Robinson M, et al. Global, regional, and national prevalence of overweight and obesity in children and adults during 1980-2013: a systematic analysis for the Global Burden of Disease Study 2013. Lancet. 2014;384(9945):766-81.

2. Kelly T, Yang W, Chen CS, et al. Global burden of obesity in 2005 and projections to 2030. Int J Obes. 2008;32(9):1431-7.

3. World Health Organization. Obesity and overweight. 2018. http:/ www.who.int/en/news-room/fact-sheets/detail/obesity-andoverweight. Accessed: 2018 DEC 01.

4. Statistica. Global gross domestic product (GDP) at current prices from 2012 to 2022 (in billion U.S. dollars). https://www.statista. com/statistics/268750/global-gross-domestic-product-gdp. Accessed: 2018 DEC 01.

5. Puzziferri N, Roshek 3rd TB, Mayo HG, et al. Long-term follow-up after bariatric surgery: a systematic review. JAMA. 2014;312(9): $934-42$.

6. Brehm BJ, Spang SE, Lattin BL, et al. The role of energy expenditure in the differential weight loss in obese women on low-fat and lowcarbohydrate diets. J Clin Endocrinol Metab. 2005;90(3):1475-82.

7. Bohmer RM. The hard work of health care transformation. N Engl J Med. 2016;375(8):709-11.

Publisher's Note Springer Nature remains neutral with regard to jurisdictional claims in published maps and institutional affiliations. 